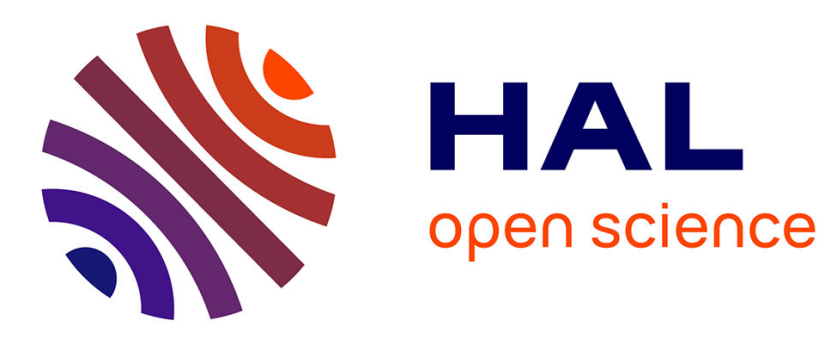

\title{
Mechanism of Breathing Transitions in Metal-Organic Frameworks
}

Carles Triguero, François-Xavier Coudert, Anne Boutin, Alain Fuchs, Alexander V Neimark

\section{- To cite this version:}

Carles Triguero, François-Xavier Coudert, Anne Boutin, Alain Fuchs, Alexander V Neimark. Mechanism of Breathing Transitions in Metal-Organic Frameworks. Journal of Physical Chemistry Letters, 2011, 2 (16), pp.2033-2037. 10.1021/jz2008769 . hal-02113294

\section{HAL Id: hal-02113294 \\ https://hal.science/hal-02113294}

Submitted on 28 Apr 2019

HAL is a multi-disciplinary open access archive for the deposit and dissemination of scientific research documents, whether they are published or not. The documents may come from teaching and research institutions in France or abroad, or from public or private research centers.
L'archive ouverte pluridisciplinaire HAL, est destinée au dépôt et à la diffusion de documents scientifiques de niveau recherche, publiés ou non, émanant des établissements d'enseignement et de recherche français ou étrangers, des laboratoires publics ou privés. 


\title{
Mechanism of Breathing Transitions in Metal-Organic Frameworks
}

\author{
Carles Triguero, ${ }^{1,{ }^{*}}$ François-Xavier Coudert, ${ }^{1, *}$ Anne Boutin, ${ }^{2}$ Alain H. Fuchs, ${ }^{1}$ Alexander V. Neimark ${ }^{1,3,{ }^{*}}$ \\ ${ }^{1}$ Chimie ParisTech \& CNRS, LECIME, Paris, France \\ ${ }^{2}$ École normale supérieure, Département de chimie, Laboratoire PASTEUR, UPMC-CNRS, Paris, France \\ ${ }^{3}$ Department of Chemical and Biochemical Engineering, Rutgers University, New Jersey, United States
}

Email:carlos-triguero@chimie-paristech.fr; fx.coudert@chimie-paristech.fr; aneimark@rutgers.edu

\begin{abstract}
We present a multiscale physical mechanism and a stochastic model of breathing transitions, which represent adsorption-induced structural transformations between large pore and narrow pore conformations in bistable metal-organic frameworks, such as MIL-53. We show that due to interplay between host framework elasticity and guest molecule adsorption, these transformations on the level of crystal occur via layer-by-layer shear. We construct a simple Hamiltonian that describes the physics of host-host and host-guest interactions and show that a respective Monte Carlo simulation model qualitatively reproduces the experimentally observed features of breathing transitions.
\end{abstract}

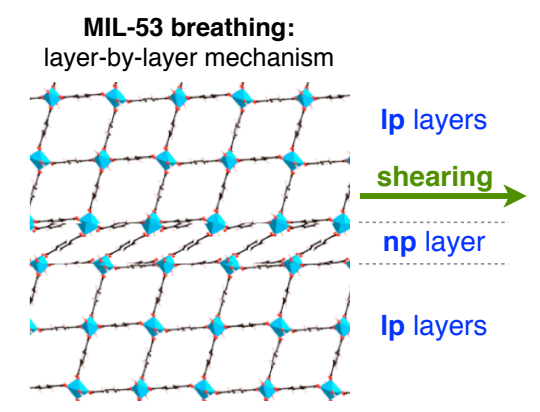

Keywords: adsorption, metal-organic frameworks, structural transitions, elasticity, Monte Carlo simulation 
Metal-organic frameworks (MOF) represent a new class of porous crystals, which has attracted physicists and material scientists worldwide due to their extraordinary physico-chemical and mechanical properties and potential applications in energy and biomedicine fields. Recent discovery of structural transformations between large pore (lp) and narrow pore (np) conformations in MOFs of MIL-53 type and others brought about a new physical phenomenon that represents a challenging problem for theoreticians $[1,2,3]$. This phenomenon called breathing is displayed in abrupt changes of the framework volume triggered by adsorption of guest molecules that is explored to devise advanced adsorbents, drug delivery systems, sensors, and actuators [4,5]. In this Letter, we suggest a multiscale cooperative mechanism of breathing transitions that involves a complex interplay of adsorption and elastic interactions on the level of crystal. This mechanism is explored with a "primitive" stochastic model of adsorption in flexible bistable frameworks that takes into account the major physical factors with the minimum input parameters and is capable of reproducing experimentally observed features. As such, we address the topical questions of the hysteretic nature of breathing transitions and the possibility of co-existence of $\mathbf{l p}$ and $\mathbf{n p}$ phases in one crystal that was highly debated in the literature recently $[15,6,7]$.

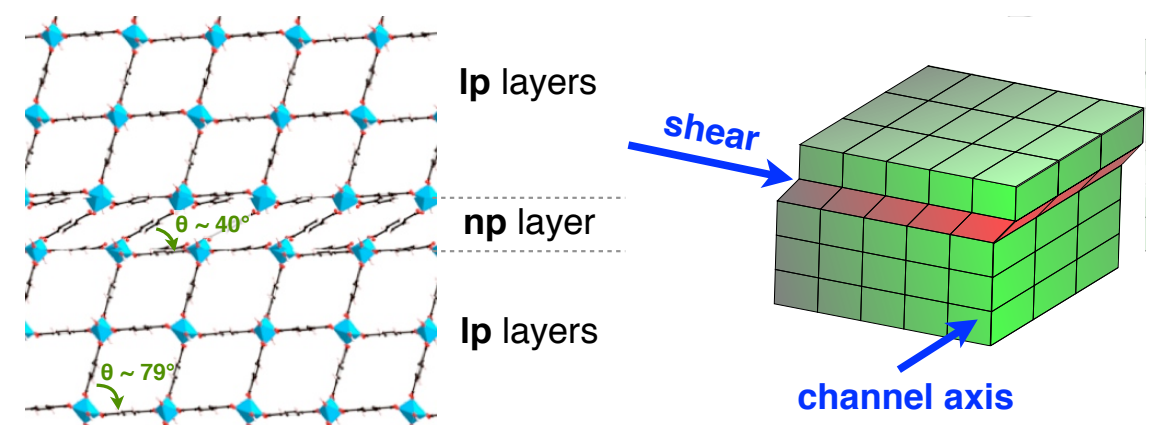

Figure 1. Schematic representation of the MIL-53 framework after the first event of Ip-np transformation, which involves in-plane shear of a $2 \mathrm{D}$ layer of cells in a direction orthogonal to the channel axis.

The most instructive examples of breathing transitions are observed in MOFs of MIL-53 family during isothermal adsorption of $\mathrm{Xe}, \mathrm{CH}_{4}, \mathrm{CO}_{2}$ and other gases [8]. MIL-53 framework is made of parallel onedimensional $\mathrm{M}(\mathrm{OH})$ chains $\left(\mathrm{M}=\mathrm{Al}^{3+}, \mathrm{Cr}^{3+}, \mathrm{Ga}^{3+}, \ldots\right)$, linked together by 1,4-benzenedicarboxylate (BDC) ligands to form linear diamond-shaped channels that are wide enough to accommodate small guest molecules. This structure may oscillate between $\mathbf{l p}$ and np phases, which have a remarkable difference in unit cell volume of up to $40 \%[9,10]$ (see schematics in Fig. 1). What is truly extraordinary is that the transition from the larger volume lp phase to the smaller volume np phase is not necessarily accompanied with the release of guest molecules that would be expected for the normal "exhaling". For example, the equilibrium state of the MIL-53 crystal at $220 \mathrm{~K}$ in the absence of guest molecules is in the lp phase, and upon Xe adsorption there first occurs the transition from unloaded lp phase to loaded np phase [11]. This transition is associated with a sharp uptake of Xe, from a loading of $\sim 0.2$ to $\sim 2.5$ molecules per unit cell, and a decrease of the crystal volume by $\sim 25 \%$. 
Upon further increase of the gas pressure, adsorption gradually proceeds in the np phase up to a certain point, when the second, now "normal", breathing transiting occurs, from the np phase to lp phase. The sample abruptly "inhales", increasing the loading from $\sim 2.7$ to $\sim 6.5$ molecules per cell and expands, compensating for the volume lost upon the first lp-np transition. On the desorption pass, the reverse normal lp-np and abnormal np-lp “exhaling” transitions take place with a prominent hysteresis.

This enigmatic breathing phenomenon is engendered by guest-host adsorption interactions mediated by the elasticity of three dimensional host framework, which are currently poorly understood. The specific variations of the linker conformations in the Ip and np phases during breathing transitions have been studied at the molecular level by Férey and co-authors, both experimentally (in-situ X-ray diffraction) [9] and using molecular simulation (single-point DFT calculations and forcefield-based dynamics) [12]. These works provide a useful insight into the chemistry of the transformation of linker bonds associated with the framework deformation. However, a knowledge gap exists between this molecular understanding and the question of how the adsorption of guest molecules induces the physical forces responsible for macroscopic expansion and contraction of the sample. The adsorption of molecules may induce a significant stress inside the elastic framework of the order of several to tens of MPa, as was recently shown [13]. The adsorption stress may be either positive or negative depending on the loading and may thus induce either framework expansion or contraction. In accord with the adsorption stress hypothesis [14], the structural transformation occurs upon achieving a certain threshold stress that the respective phase cannot withhold. Although this hypothesis describes on a semi-quantitative level the breathing transitions driven by $\mathrm{Xe}$ and $\mathrm{CH}_{4}$ adsorption on MIL-53(Al) [14,15], it is macroscopic in nature and does not shed light on the mechanism and dynamics of structural transformations on the unit cell level. As shown below, adsorption interactions in the elastic framework are long range and bring about a cooperative mechanism of cell filling: adsorption in one cell triggers adsorption in the 1D channel, which this cell belongs to, and this, in turn, triggers adsorption and the respective phase transformation in the whole 2D layer of channels.

From the geometrical standpoint, the Ip and np phases of MIL-53 differ mainly by the shape of cells, which can be characterized by the angle $\theta$ of its rhombus cross-section; in lp phase, with a pore diameter of $9.5 \AA$, $\theta \sim 79^{\circ}$, while in $\mathbf{n p}$ phase (with pore diameter of $3.5 \AA$ ) $\theta \sim 40^{\circ}$ (Fig. 1). At the same time, the variation in the linker lengths between the two phases is of the order of $0.4 \AA$ (just only 3.8\%), making the length of the rhombus sides essentially invariant upon deformation of the structure. This strong constraint on the framework geometry plays a crucial role in the dynamics of the phase transformation, as the deformation of a single cell can be quantified by just one degree of freedom represented by the angle $\theta$. The assumption of the preserved rhombus shape with the fixed length of sides imposes a strong correlation on the deformation of neighboring cells in order to ensure the lattice integrity. First, the cells along a channel must coherently deform, as two stacked rhombuses cannot match unless they have the same angle $\theta$. Secondly, the channels connected by sides of fixed length must possess equal $\theta$ along the shear direction. This defines a 2D layer of cells inside which all the channels have the same cross section. Mismatches in the rhombus angle can exist along the direction perpendicular to the shear plane. Thus, the only possible mechanism of framework deformation is the layer-by-layer shear and the 
elementary deformation consists of the shear of the layer of cells in the direction perpendicular to the channel axis (represented in Fig. 1). This conclusion can be formally derived from the Saint-Vénant principle by writing the equations for the elastic compatibility conditions linking the strain in neighboring cells [16]. The elastic compatibility equations yield a long-range interaction, similar to that of martensitic transformations [17]. In the case of the MIL-53 structure, the long-range interaction works as a rigid constraint requiring the similitude of the cell shapes within the 2D layer. This means that the phase transformations occur in a cooperative manner and necessarily involve the entire layer of cells, all of which must be in the same phase. As a consequence, the layer of cells, rather than the single cell, can be taken as a base unit of the framework mechanical model. The 3D framework is thus presented as a stack of 2D layers of identical cells that makes possible to formulate a onedimensional minimalistic model of the framework deformation that captures the main system properties with a minimum number of input parameters.

Within our primitive 1D model, two main variables describe the state of each cell layer at given external thermodynamic conditions. First, we quantify the cell deformation by one geometrical parameter, the symmetrized strain $e$, which is derived from discrete elasticity theory [18] by renormalization of the shear deformation strain so that the stable lp and $\mathbf{n p}$ structures correspond to $e=-1$ and $e=+1$ respectively. The second variable is the adsorption loading $n$, or the mean number of guest molecules per unit cell in the layer. By averaging the loading over the whole framework as a function of the gas external pressure (or chemical potential), one obtains the adsorption isotherm. This quantity is the key observable of the system, as it is measured in isothermal adsorption experiments. The experimental adsorption isotherms typically display two sharp yet continuous transitions from empty lp phase to almost fully loaded np phase and from np phase to fully loaded lp phase. The loading capacities of both phases differ significantly and represent the main quantitative parameters determining the adsorption isotherm behavior. Our primitive model is discrete; it allows for adsorption of either 0,1 , or 2 molecules in the cell, that corresponds schematically to empty cell, np cell capacity, and Ip cell capacity. This simplification can be easily generalized to introduce additional loading levels, or to describe adsorption by a continuous variable.

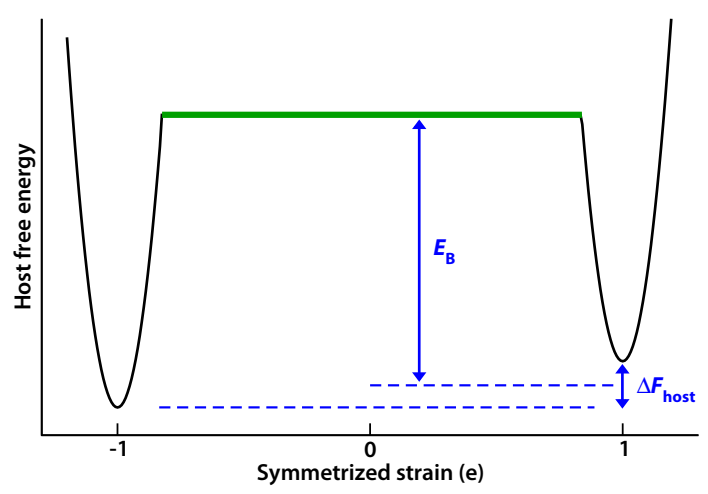

Figure 2: Schematics of the host free energy landscape for the "dry" bistable framework, as a function of symmetrized strain $e$. Two regions of elastic deformation around the equilibrium lp and $\mathbf{n p}$ structures $(e= \pm 1)$ 
are approximated by parabolas. The non-elastic region in-between is taken into account by introducing a free energy barrier $E_{\mathrm{B}}$ in the dynamic model.

The proposed model describes the interplay between guest adsorption and host framework deformation in terms of a Hamiltonian that depends on the loading $\mathbf{n}$ and strain $\mathbf{e}$ fields and is expressed per unit cell,

$$
H(\mathrm{n}, \mathrm{e})=\sum_{i}\left[\frac{c_{0}}{2}\left(e_{i}-s_{i}\right)^{2}+\frac{\Delta F}{2} s_{i}+\frac{c_{1}}{2}\left(\nabla e_{i}\right)^{2}+\varepsilon\left(n_{i}, s_{i}\right)-\sigma_{a}\left(n_{i}, s_{i}\right)\left(e_{i}-s_{i}\right)\right] .
$$

The first term in the right-hand side accounts for elastic deformation of individual cells with the local elastic energy is modeled as a biparabolic potential [19], see Fig. $2 ; s_{i}=\operatorname{sgn}\left(\mathrm{e}_{i}\right)$ denotes the phase of the cell $\left(s_{i}=-1\right.$ in Ip phase and $s_{i}=+1$ in $\mathbf{n p}$ phase), and $\left(e_{i}-s_{i}\right)$ is the local deformation from the reference state of the respective phase. The effective elastic constant $c_{0}$ is taken equal for both phases. The second term $\Delta F$ represents the difference in the free energy between empty $\mathbf{n p}$ and empty $\mathbf{l p}$ phases, which is positive, reflecting the fact that the initial "dry" state corresponds to the stable lp phase. The third term corresponds to the non-local cell-cell elastic energy proportional to the strain gradient squared with $\nabla e_{i}=e_{i+1}-e_{i}$; it penalizes the formation of interfaces between $\mathbf{l p}$ and $\mathbf{n p}$ phases by the interfacial energy of $2 c_{1}$, and also levels elastic deformations in neighboring cells of the same phase. The fourth and fifth terms determine the host-guest interactions as the energy of adsorption $\varepsilon\left(n_{i}, s_{i}\right)$ at given loading $n_{i}$ and deformation $e_{i}$ expanded around the adsorption energy $\varepsilon\left(n_{i}, e_{i}\right)$ in non-deformed reference $\mathbf{l p}$ or $\mathbf{n p}$ state, $e_{i}=s_{i}$. This expression gives rise to a quantity of prominent physical significance: the adsorption stress induced on the host framework due to its interactions with the guest molecules, which is defined as

$$
\sigma_{a}\left(n_{i}, s_{i}\right)=-\partial \varepsilon\left(n_{i}, e\right) /\left.\partial e\right|_{e=s_{i}, n_{i}},
$$

in line with the thermodynamic definition of the adsorption stress [12,20,21]. The adsorption stress couples the host-guest interactions with the elastic deformation and accounts for the forces exerted by the guest molecules on the host framework. It can be either negative or positive depending on the loading and thus cause either elastic contraction or expansion. As such, the number of input parameters characterizing adsorption in our model is reduced to 8: 4 adsorption energies $\varepsilon\left(n_{i}, e_{i}\right)$ and 4 adsorption stresses $\sigma_{a}\left(n_{i}, s_{i}\right), n_{i}=1$ or 2 , and $s_{i}= \pm 1$.

Modeling the dynamics of the coupled adsorption and deformation in the process of incremental stepwise variation of the chemical potential of the adsorbed gas, we make a further assumption that the local elastic relaxation of the framework occurs on a much smaller time scale than the establishment of adsorption equilibrium at a given chemical potential. This assumption allows us to determine the continuous elastic strain field $\mathbf{e}$ as a function of the discrete fields $\mathbf{s}$ and $\mathbf{n}$ by minimizing the Hamiltonian (1) with respect to $\mathbf{e}$, $\partial H /\left.\partial \mathbf{e}\right|_{\mathbf{n}, \mathbf{s}}=0$. Due to the quadratic nature of the elastic potential, this minimization yields a system of linear equations, which are easily solved. As such, we discretize the model and each cell layer is characterized by its state variables $s_{i}= \pm 1$ and loading $n_{i}=0,1$, or 2 , with the Hamiltonian being a function $H(\mathbf{n}, \mathbf{s})$. 
In mimicking the adsorption process, we performed grand canonical Monte Carlo (MC) simulations of the 1D array of cell layers with periodic boundary conditions. In this molecular simulation, each element in the 1D system is a layer of MIL-53 unit cells; no atomistic detail of the framework is included. The whole system is in contact with a fictitious gas reservoir. The thermodynamic variables are the size of the system $L$, the temperature $T$ and the chemical potential $\mu$ of the adsorbing gas. Two kinds of MC moves are considered, which lead to the change of the cell state: adsorption or desorption of one particle $\left(n_{i} \rightarrow n_{i} \pm 1\right)$ and the phase switch between Ip and np structures $\left(s_{i} \rightarrow-s_{i}\right)$. The exponential in the acceptance probabilities for particle insertions and deletions moves in the standard Metropolis scheme is written as $\exp \left[-\beta\left(H\left(\left\{n_{i \pm 1}, n_{j \neq i}, \mathrm{~s}_{j}\right\}\right)-H\left(\left\{n_{j}, \mathrm{~s}_{j}\right\}\right) \pm \mu\right)\right]$. The probability of the phase switch includes the energy landscape of the host (1) as a biparabolic function with two very narrow wells, separated by a wide region where the behavior of the system is not elastic. We approximated this "no man's land" region of energy, which is not sampled in experiments, by an energy barrier $E_{\mathrm{B}}$ (green horizontal line on Fig. 2) following the scheme employed by Kang et al. [22] in studies of activated diffusion. As such, we introduced the energy barrier directly in the acceptance probabilities of the phase change, which becomes proportional to $\exp \left[-\beta\left(H\left(\left\{n_{j},-\mathrm{s}_{i}, s_{j \neq i}\right\}\right)-H\left(\left\{n_{j}, \mathrm{~s}_{j}\right\}\right)+E_{\mathrm{B}} / 2\right)\right]$ if $\Delta H \leq 2 E_{\mathrm{B}}$.

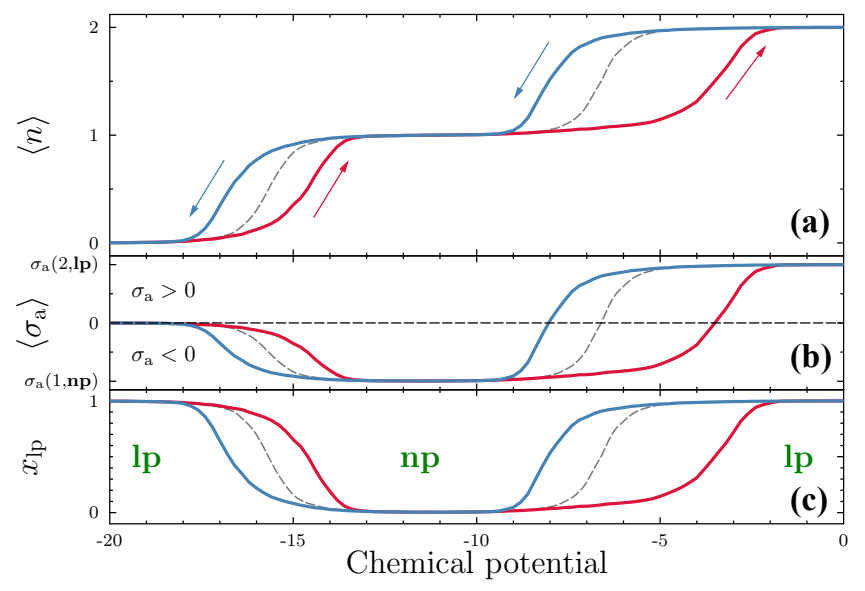

Figure 3: Breathing transitions during the adsorption-desorption cycle. (a) Adsorption isotherm, (b) adsorption stress, and (c) lp phase composition as a function of the chemical potential. Adsorption (red line), desorption (blue line), reversible isotherm obtained by ignoring the energy barrier $E_{\mathrm{B}}$ in simulation (gray). Model parameters from Table 1, coupling parameter $c_{1}=1$.

\begin{tabular}{cccc}
\hline$c_{0}=100$ & $L=2000$ & $E_{\mathrm{B}}=10.5$ & $\Delta F_{\text {host }}=5.0$ \\
$\varepsilon(1, \mathbf{l p})=0$ & $\varepsilon(1, \mathbf{n p})=-10$ & $\varepsilon(2, \mathbf{l p})=-2$ & $\varepsilon(1, \mathbf{n p})=3$ \\
$\sigma_{\mathrm{a}}(1, \mathbf{l p})=-10$ & $\sigma_{\mathrm{a}}(1, \mathbf{n p})=-10$ & $\sigma_{\mathrm{a}}(2, \mathbf{l} \mathbf{p})=10$ & $\sigma_{\mathrm{a}}(1, \mathbf{n p})=10$ \\
\hline
\end{tabular}

Table 1: Values of the model parameters used in this work. System size is adimensional, other parameters are given in units of $k_{\mathrm{B}} T$. 
As the result of simulation, we determined the experimentally measurable quantities: the adsorption isotherm as the average loading, the sample deformation through the phase composition, and the strain field, all as functions of the gas chemical potential. The set of model parameters is given in Table 1. These parameters were chosen in order to reproduce qualitatively some of the known characteristics of Xe adsorption in MIL53(Al)[11]: transition pressures, magnitude of adsorption-induced elastic deformation, and free energy difference between the phases. The coupling parameter $c_{1}$ was varied from 0 to 4 . The adsorption-desorption isotherm $\langle n\rangle$, obtained by gradually increasing and then decreasing the gas chemical potential, are reported in Fig. 3, along with the respective variation of phase composition $x_{\mathrm{lp}}$ and adsorption-induced stress $\left\langle\sigma_{\mathrm{a}}>\right.$. The calculated stepwise isotherm with two hysteresis loops parallels the experimental isotherm [11]. The phase composition curve is also comparable to the one calculated using an earlier model [14]. Each MC simulation run was 30,000 steps. The length of the simulation run plays a role of the equilibration time in adsorption experiments. While we ensured the establishment of adsorption equilibrium on the cell level, the phase transformation represents a rare event and, as in experiments, occurs in a hysteretic fashion. It is worth noting that it was our goal to reproduce the hysteretic nature of breathing transitions in our simulations rather than to avoid the metastable states and to confine ourselves on the conditions of phase equilibrium. This is why we introduce the energy barrier $E_{\mathrm{B}}$ in the phase change acceptance probabilities. The longer the run the larger is the probability to cross the energy barrier associated with the phase change. Without this barrier, the system would follow equilibrium configurations and the adsorption isotherms were reversible, as shown in Fig. 3. For a given simulation length, the width of the hysteresis loop sharply increases with the height of the energy barrier.
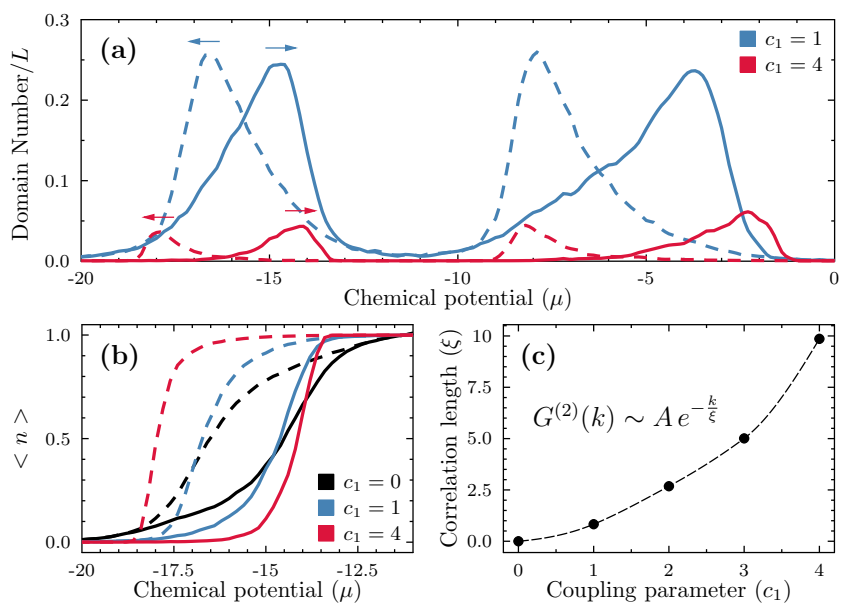

Figure 4: Effect of cell-cell coupling. (a) Number of domains in the system as a function of $\mu$ for $c_{1}=1$ (blue), and $c_{1}=4$ (red), upon adsorption (solid) and desorption (dotted). (b) Adsorption hysteresis loop for the lowpressure lp-np transition, for different values of $c_{1}$. (c) Variation of the correlation length $\xi$ with the coupling parameter $c_{1}$.

The proposed model allowed us to study different dynamics of the lp-np transformations on the level of the whole framework. For a given set of model parameters, the strength of the lp-np interfacial energy $2 c_{1}$ 
determines the pathway on the phase transformations in the course of the adsorption-desorption cycle. Two different dynamic regimes are observed depending on the coupling parameter $c_{1}$. In the uncorrelated limit, $c_{1} \rightarrow 0$, the Hamiltonian becomes fully local; the cell layers do not interact, and the phase transformations within the individual layers occur at random. The adsorption isotherm then shows relatively smooth phase transformations (see black hysteresis loop in Fig. 4b). In this limit, it is easier to overcome the energy barrier $E_{\mathrm{B}}$ due to thermal fluctuations. In contrast, the existence of an interfacial energy effectively increases the energy barrier of the phase change of an isolated layer in a domain. As such, the phase transformation occurs in an avalanche manner: once the first nucleation event consisting of lp-np transformation in one layer happens, the further lp-np transformations will most likely proceed in the neighboring layers. This prevents microstructuration, leading to a small number of large domains (Fig. 4a) and sharper adsorption and desorption hysteresis loops (blue and red loop in Fig. 4b). Furthermore, the parameter $c_{1}$ is directly linked with the system correlation length, $\xi$ [23], as shown in Fig. 4c. In the limit of large $c_{1}$, $\xi$ diverges and only one domain would grow since the phase coexistence is strictly prohibited.

In conclusions, the elastic compatibility conditions on the level of crystal imply that the elementary lp-np transformation in the bistable MIL-53 structure necessarily involves the shear of the entire layer of unit cells and proceeds in either an avalanche and random manner. While our dynamic model predicts the possibility of phase co-existence in the process of Ip-np transformation, additional information about cell-cell correlations is required to determine to which extent the experimental systems may follow this scenario. This information might come from space-resolved experimental technique showing the evolution of the cell parameters near an lp-np interface (if it exists), or from first-principle atomistic calculations of the elastic constants of lp and np phases. The suggested model can be upgraded to include realistic adsorption capacities of the $\mathbf{l p}$ and $\mathbf{n p}$ phases for a given guest-host system and further expanded to tree-dimensional anisotropic structures.

A.V.N. and C.T. acknowledge support from the Blaise Pascal International Chair award.

\section{References}

(1) Férey, G.; Serre, C. Large Breathing Effects in Three-Dimensional Porous Hybrid Matter: Facts, Analyses, Rules and Consequences Chem. Soc. Rev. 2009, 38, 1380-1399.

(2) Horike, S.; Shimomura, S.; Kitagawa, S. Soft Porous Crystals. Nat. Chem. 2009, 1, 695-704.

(3) Coudert, F.-X.; Boutin, A.; Jeffroy, M.; Mellot-Draznieks, C.; Fuchs, A.H. Thermodynamic Methods and Models to Study Flexible Metal-Organic Frameworks. Chem. Phys. Chem. 2011, 12, 247-258.

(4) Liu, Y.; Her, J.-H.; Dailly, A.; Ramirez-Cuesta, A. J.; Neumann, D. A.; Brown, C. M. Reversible Structural Transition in MIL-53 with Large Temperature Hysteresis. J. Am. Chem. Soc. 2008, 130, 11813-11818.

(5) Beurroies, I.; Boulhout, M.; Llewellyn, P. L.; Kuchta, B.; Férey, G.; Serre, C.; Denoyel, R. Using Pressure to Provoke the Structural Transition of Metal-Organic Frameworks. Angew. Chem. Int. Ed. 2010, 49, 75267529. 
(6) Millange, F.; Serre, C.; Guillou, N.; Férey, G.; Walton, R. I. Structural Effects of Solvents on the Breathing of Metal-Organic Frameworks: an In Situ Diffraction Study. Angew. Chem. Int. Ed. 2008, 47, 4100.

(7) Springuel-Huet, M.-A.; Nossov, A.; Adem, Z.; Guenneau, F.; Volkringer, C.; Loiseau, T.; Férey, G.; Gédéon, A. ${ }^{129}$ Xe NMR Study of the Framework Flexibility of the Porous Hybrid MIL-53(Al). J. Am. Chem. Soc. 2010, 132, 11599-11607.

(8) Coudert, F.-X.; Jeffroy, M.; Fuchs, A. H.; Boutin, A.; Mellot-Draznieks, C. Thermodynamics of GuestInduced Structural Transitions in Hybrid Organic-Inorganic Frameworks. J. Am. Chem. Soc. 2008, 130, 14294-14302.

(9) Serre, C.; Bourrelly, S.; Vimont, A.; Ramsahye, N. A.; Maurin, G.; Llewellyn, P. L.; Daturi, M.; Filinchuk, Y.; Leynaud, O.; Barnes, P.; Férey, G. An Explanation for the Very Large Breathing Effect of a MetalOrganic Framework during $\mathrm{CO}_{2}$ Adsorption. Adv. Mater. 2007, 19, 2246.

(10) Serre, C.; Millange, F.; Thouvenot, C.; Noguès, M.; Marsolier, G.; Louër, D.; Férey, G. Very Large Breathing Effect in the First Nanoporous Chromium(III)-Based Solids. J. Am. Chem. Soc. 2002, 124, 13519-13526.

(11) Boutin, A.; Springuel-Huet, M.-A.; Nossov, A.; Gédéon, A.; Loiseau, T.; Volkringer, C.; Férey, G.; Coudert, F.-X.; Fuchs, A.H. Breathing Transitions in MIL-53(Al) Metal-Organic Framework upon Xenon Adsorption. Angew. Chem. Int. Ed. 2008, 48, 8314-8317.

(12) Salles, F.; Ghoufi, A.; Maurin, G.; Bell, R.G.; Mellot-Draznieks, C.; Férey, G. Molecular Dynamics Simulations of Breathing MOFs: Structural Transformations of MIL-53(Cr) upon Thermal Activation and $\mathrm{CO}_{2}$ Adsorption. Angew. Chem. Int. Ed. 2008, 47, 8487-8491.

(13) Neimark, A.V.; Coudert, F.-X.; Triguero, C.; Boutin, A.; Fuchs, A.H.; Beurroies, I.; Denoyel, R. Structural Transitions in MIL-53 (Cr): View from Outside and Inside. Langmuir, 2011, 27, 4734.

(14) Neimark, A.V.; Coudert, F.-X.; Boutin, A.; Fuchs, A.H. Stress-Based Model for the Breathing of MetalOrganic Frameworks. J. Phys. Chem. Lett. 2010, 1, 445.

(15) Boutin, A.; Coudert, F.-X.; Springuel-Huet, M.-A.; Neimark, A. V.; Férey, G.; Fuchs, A. H. The Behavior of Flexible MIL-53(Al) upon $\mathrm{CH}_{4}$ and $\mathrm{CO}_{2}$ Adsorption. J. Phys. Chem. C 2010, 114, 22237-22244.

(16) Shenoy, S. R. ; Lookman, T. ; Saxena, A. ; Bishop, A.R. Martensitic textures: Multiscale Consequences of Elastic Compatibility. Phys. Rev. B 1999, 60, R12537.

(17)-Rasmussen, K. O.; Lookman, T.; Saxena, A.; Bishop, A.R.; Albers, R. C.; Shenoy, S.R. Three-Dimensional Elastic Compatibility and Varieties of Twins in Martensites. Phys. Rev. Lett. 2001, 87, 055704.

(18) Ariza, M. P.; Ortiz, M. Discrete Crystal Elasticity and Discrete Dislocations in Crystals. Arch. Rat. Mech. Anal. 2005, 178, 149 (2005).

(19) Müller, I.; Villaggio, P. Model for an Elastic-Plastic Body. Arch. Rat. Mech. Anal. 1977, 65, 25-46.

(20) Ravikovitch, P. I.; Neimark, A. V.; Density Functional Theory Model of Adsorption Deformation. Langmuir 2006, 22, 10864. 
(21) Kowalczyk, P.; Ciach, A.; Neimark, A.V. Adsorption-Induced Deformation of Microporous Carbons: Pore Size Distribution Effect. Langmuir 2008, 24, 6603.

(22) Kang, H. C.; Weinberg, W. H. Dynamic Monte Carlo with a Proper Energy Barrier: Surface Diffusion and Two-Dimensional Domain Ordering. J. Chem. Phys. 1989, 90, 2824.

(23) The correlation length $\xi$ is obtained by fitting the correlation function as $G^{(2)}(k) \sim A \exp [-k / \xi]$. 